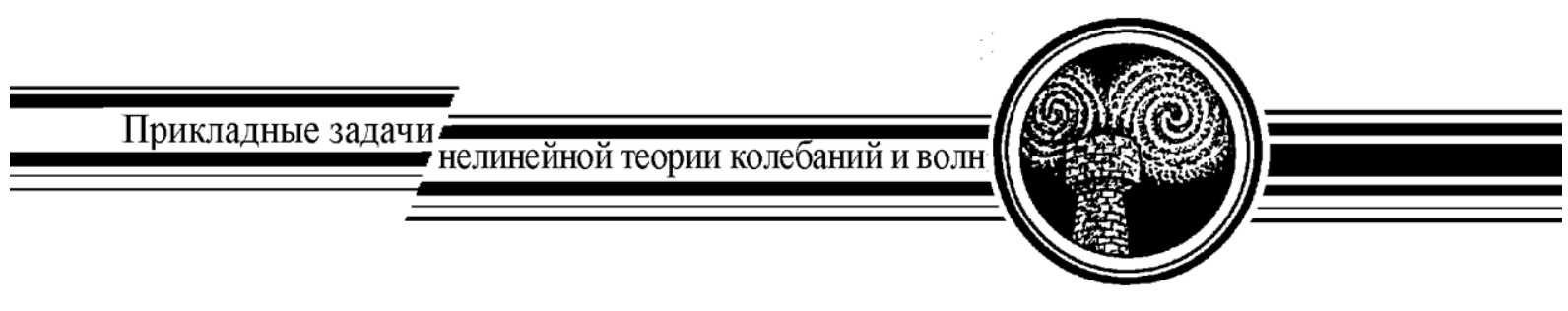

УДК 530.182;517.9

https://doi.org/10.18500/0869-6632-2019-27-3-86-98

\title{
Динамика слабодиссипативной автоколебательной системы под внешним импульсным воздействием с амплитудой, полиномиально зависящей от динамической переменной
}

\author{
А. В. Голоколенов \\ Саратовский национальный исследовательский \\ государственный университет имени Н.Г. Чернышевского \\ Россия, 410012 Саратов, Астраханская, 83 \\ E-mail: golokolenovav@gmail.com \\ Поступила в редакиию 01.03.2019; принята к публикации 14.05.2019
}

\begin{abstract}
Тема и цель. В работе исследуется динамика осциллятора ван дер Поля под импульсным воздействием, амплитуда которого зависит нелинейным образом от динамической переменной. В качестве функций, описывающих эту зависимость, выбираются разложения функции $\cos x$ в ряд Тейлора вблизи нуля. Известно, что в случае, когда зависимость амплитуды внешнего воздействия от динамической переменной описывается квадратичным полиномом, такая система демонстрирует наличие критической точки гамильтоновского типа, а при выборе зависимости в виде $\cos x$ - стохастической паутины в консервативном пределе. Исследованные модели. Исследование проводится для исходной потоковой системы и для приближенного дискретного отображения. Результаты. Исследованы изменения устройства пространства параметров и фазового пространства при изменении вида функции внешнего воздействия. Показано, что усложнение вида функции приводит к увеличению количества седло-узловых бифуркаций, происходящих в системе при уменьшении параметра диссипации.
\end{abstract}

Ключевые слова: нелинейная динамика, седло-узловая бифуркация, стохастическая паутина, показатель Ляпунова, мультистабильность.

Образец цитирования: Голоколенов А.В. Динамика слабодиссипативной автоколебательной системы под внешним импульсным воздействием с амплитудой, полиномиально зависящей от динамической переменной // Известия вузов. ПНД. 2019. Т. 27, № 3. С. 86-98. https://doi.org/10.18500/0869-6632-2019-27-3-86-98 


\title{
Dynamics of weakly dissipative self-oscillatory system at external pulse influence, which amplitude is depending polynomially on the dynamic variable
}

\author{
A. V. Golokolenov \\ Saratov State University \\ 83, Astrakhanskaya str., 410012 Saratov, Russia \\ E-mail: golokolenovav@gmail.com \\ Received 01.03.2019; accepted for publication 14.05.2019
}

\begin{abstract}
Topic and aim. In this work, we study the dynamics of the kicked van der Pol oscillator with the amplitude of kicks depending nonlinearly on the dynamic variable. We choose the expansions of the function $\cos x$ in a Taylor series near zero, as functions describing this dependence. It is known that such a system demonstrates the existence of a Hamiltonian-type critical point in the case when the dependence of the amplitude of an external force on a dynamic variable is described by a quadratic polynomial, and when choosing a dependence in the form of $\cos x$ - a stochastic web in the conservative limit. Investigated models. The investigation is conducted for the original flow system and for an approximate discrete mapping. Results. We have investigated the changes in the structure of the parameter space and the phase space when changing the form of the function of external force. It is shown that the complication of the form of the function leads to an increase in the number of saddle-node bifurcations occurring in the system with a decrease in the dissipation parameter.
\end{abstract}

Key words: nonlinear dynamics, saddle-node bifurcation, stochastic web, Lyapunov exponent, multistability.

Reference: Golokolenov A.V. Dynamics of weakly dissipative self-oscillatory system at external pulse influence, which amplitude is depending polynomially on the dynamic variable. Izvestiya VUZ, Applied Nonlinear Dynamics, 2019, vol. 27, no. 3, pp. 86-98. https://doi.org/10.18500/0869-6632-2019-27-3-86-98

\section{Введение}

Известно, что динамика консервативных и диссипативных нелинейных систем довольно сильно различается. Например, различны закономерности, наблюдающиеся при переходе к хаосу через каскад бифуркаций удвоения периода - структура пространства параметров в окрестности критической точки характеризуется различными скейлинговыми константами $[1,2]$. В силу невозможности полного исключения диссипации в любой реально функционирующей системе физическая реализация системы со строго консервативной динамикой представляется невозможной. Так, например, известно, что появление в изначально консервативной системе даже малых постоянных потерь энергии приводит к тому, что при приближении системы к критической точке перехода к хаосу динамика такой системы начинает подчиняться закономерностям, характерным для диссипативных систем [3]. В связи с этим исследование динамики систем, полученных из консервативных путем добавления в них малых диссипативных возмущений, вызывает определённый интересе с точки зрения как математики (см., например, монографию [4]), так и физики в частности, радиофизики, теории колебаний и нелинейной динамики. В этой области можно выделить несколько крупных направлений.

Одно из них связано с изучением критического поведения на границе хаоса. Поскольку, как отмечалось выше, в случае наличия в системе хотя бы малой постоянной диссипации реализация характерного для консервативных систем типа критического поведения на границе хаоса невозможна, невозможно и его наблюдение в физически реализуемых системах такого класса. Если всё же задаться целью сконструировать нелинейную систему с гамильтоновским

Голоколенов А.B. 
критическим поведением, допускающую физическую реализацию, естественным будет обращение к классу систем с переменным уровнем диссипации. Идея при этом состоит в том, чтобы определённым образом «компенсировать» потери энергии специальным образом подобранным внешним воздействием [5]. Так, например, была сконструирована такая система на базе осциллятора ван дер Поля под внешним импульсным воздействием, зависимость амплитуды которого от динамической переменной описывается квадратичным полиномом [6]. Было произведено исследование устройства пространства параметров и фазового пространства такой системы $[7,8]$, в частности, было показано существование в приближенной дискретной модели такой системы гамильтоновской критической точки (точки Н-типа) как феномена коразмерности 2 в широком диапазоне параметров. Другим важным направлением исследований динамики систем с малым уровнем диссипации является изучение сильной мультистабильности, возникающей в таких системах при приближении уровня диссипации к нулю. Сосуществование большого числа низкопериодических аттракторов было впервые показано для довольно простой слабодиссипативной модели - так называемого «отображения ротора» [9]. К настоящему моменту существует множество работ, посвящённых изучению мультистабильности и закономерностей сосуществования аттракторов в различных нелинейных системах с малой диссипацией, как модельных, так и имеющих физическую мотивацию (см., например, [10-20]). В то же время следует отметить, что во всех перечисленных работах речь идёт о динамике систем, полученных добавлением постоянной (то есть не зависящей от динамических переменных системы) диссипации в консервативные модели, удовлетворяющие условию КАМ-теоремы [21]. Логичным развитием этого направления исследований является распространение рассмотрения на системы, консервативная версия которых является с точки зрения КАМ-теоремы вырожденной. Одной из наиболее простых и известных моделей такого рода является так называемая система-генератор «стохастической паутины», предложенная Заславским [22]. Эта система может быть записана в виде консервативного линейного осциллятора под внешним импульсным периодическим воздействием, зависимость амплитуды которого от обобщённой координаты осциллятора представляет собой гармоническую функцию. Динамика такой системы в слабо диссипативном случае рассматривалась ранее как для случая введения линейной малой диссипации [23], так и при добавлении в систему малой диссипации «автоколебательного» типа [24,25]. В последнем случае исследуемая система фактически представляет собой находящийся под внешним импульсным воздействием осциллятор ван дер Поля с малыми значениями параметров, отвечающих за линейную и нелинейную диссипацию.

Таким образом, можно заметить, что одна и та же модель - осциллятор ван дер Поля под периодическим импульсным воздействием переменной амплитуды - способна демонстрировать феномены, относящиеся к различным направлениям исследований при выборе зависимости амплитуды внешнего воздействия от обобщённой координаты осциллятора в виде квадратичной либо гармонической функции. В связи с этим кажется интересным рассмотреть динамику моделей, занимающих «промежуточное» положение между двумя этими случаями. Для этой цели можно, например, рассматривать модели, получаемые при выборе гармонической функции в виде косинуса и разложении её в ряд Тейлора в окрестности нуля - самой простой из них, очевидно, будет как раз модель с квадратичной функцией амплитуды. В этом случае можно получить «иерархию» моделей с функциями амплитуды импульсного воздействия в виде полиномов чётных степеней и проследить за эволюцией устройства фазового пространства и пространства параметров таких систем при изменении порядка разложения.

В настоящей работе рассматриваются модели, полученные при разложении косинуса в ряд до 2 и 4 степени. В разделе 1 описывается исследуемая система и рассматривается устройство её пространства параметров при вариации функции внешнего воздействия, в разделе 2 приводятся результаты исследования фазового пространства. 


\section{1. Исследуемая система и устройство её пространства параметров}

Уравнение исследуемой системы имеет вид

$$
\ddot{x}-\left(\gamma-\mu x^{2}\right) \dot{x}+\omega_{0} x=\sum_{m=-\infty}^{\infty} F(x) \delta(t-n T) .
$$

Здесь $\gamma$ и $\mu$ - параметры линейной и нелинейной диссипации, соответственно, при этом отрицательные значения $\gamma$ соответствуют положительной линейной диссипации, то есть наличию в автономной системе устойчивой неподвижной точки. Частота внешнего импульсного воздействия $2 \pi / T$ в 4 раза больше собственной частоты автономной системы $\omega_{0}$. Исследование динамики системы проводилось для трех видов функций внешнего воздействия, а именно $F(x)=\lambda \cos x$ и полиномов, представляющих собой разложение косинуса в ряд Тейлора вблизи нуля $F(x)=\lambda\left(1-x^{2} / 2\right)$ и $F(x)=\lambda\left(1-x^{2} / 2+x^{4} / 24\right)$. При численном моделировании производилось решение автономного уравнения в промежутках между импульсами методом Рунге-Кутты 4 порядка с шагом, составлявшим 0.01 периода внешнего воздействия. Также, решая автономное уравнение в промежутках между импульсами методом медленно меняющихся амплитуд, можно получить для такой системы приближенное дискретное отображение [6]

$$
x_{n+1}=B \frac{F\left(x_{n}\right)+y_{n}}{\sqrt{1+C\left[x_{n}^{2}+F\left(x_{n}+y_{n}\right)^{2}\right]}}, \quad y_{n+1}=-B \frac{x_{n}}{\sqrt{1+C\left[x_{n}^{2}+F\left(x_{n}+y_{n}\right)^{2}\right]}},
$$

где параметр $C$ определяет нелинейную диссипацию, $B<1$ соответствует положительной линейной диссипации, $B>1$ - отрицательной. В случае выбора квадратичной функции амплитуды система перенормировкой переменных и параметров сводится к изученной в [6-8], и следует ожидать качественного совпадения структуры пространства параметров. Будем рассматривать динамику системы на плоскости $(\lambda, \gamma)$ для потоковой системы $(1)$ и $(\lambda, B)$ для отображения (2), зафиксировав параметр нелинейной диссипации. На рис. 1 приведены карты ляпуновских показателей для систем с различными функциями внешнего воздействия.

На картах присутствуют области периодической, квазипериодической и хаотической динамики, и можно видеть различные сценарии перехода к хаосу: через каскад бифуркаций удвоения периода при увеличении параметра $\lambda$ в области отрицательных значений $\gamma$, а также через разрушение квазипериодической динамики и, соответственно, через каскад бифуркаций удвоения периода внутри языков синхронизации в окрестности линии $\lambda=0$ в области $\gamma>0(B>1)$. При этом для функции $F(x)=\lambda\left(1-x^{2} / 2\right)$ (рис. $\left.1, a, b\right)$ области хаотической динамики, возникшие через эти сценарии, на карте разделены. На границе между ними находится критическая точка Н-типа (рис. $1, b$ ), которую можно найти как предел последовательности точек, в которых заканчиваются линии бифуркаций удвоения периода (или, говоря иначе, их терминальных точек). Непосредственно для данной системы положение критической точки не определялось, но, поскольку, как уже говорилось выше, рассматриваемая система эквивалентна с точностью до замены переменных системе, рассмотренной в [6-8], о наличии критической точки Н-типа в отображении можно говорить с уверенностью. На карте ляпуновских показателей для системы с функцией внешнего воздействия вида $F(x)=\lambda\left(1-x^{2} / 2+x^{4} / 24\right)$ (рис. $1, c, d$ ) видно, как трансформируется плоскость параметров: происходит расширение хаотической и квазипериодической областей и сужение языков синхронизации вдоль оси $\lambda$. Разделённые ранее области хаоса, возникшего двумя разными путями, теперь оказываются соединены. В частности, видно, что область предполагаемого местонахождения критической точки Н-типа, находившаяся ранее на границе между двумя этими хаотическими областями и областью разбегания траекторий на бесконечность, теперь находится просто на границе общей области с хаотической динамикой.

Голоколенов А.B. 

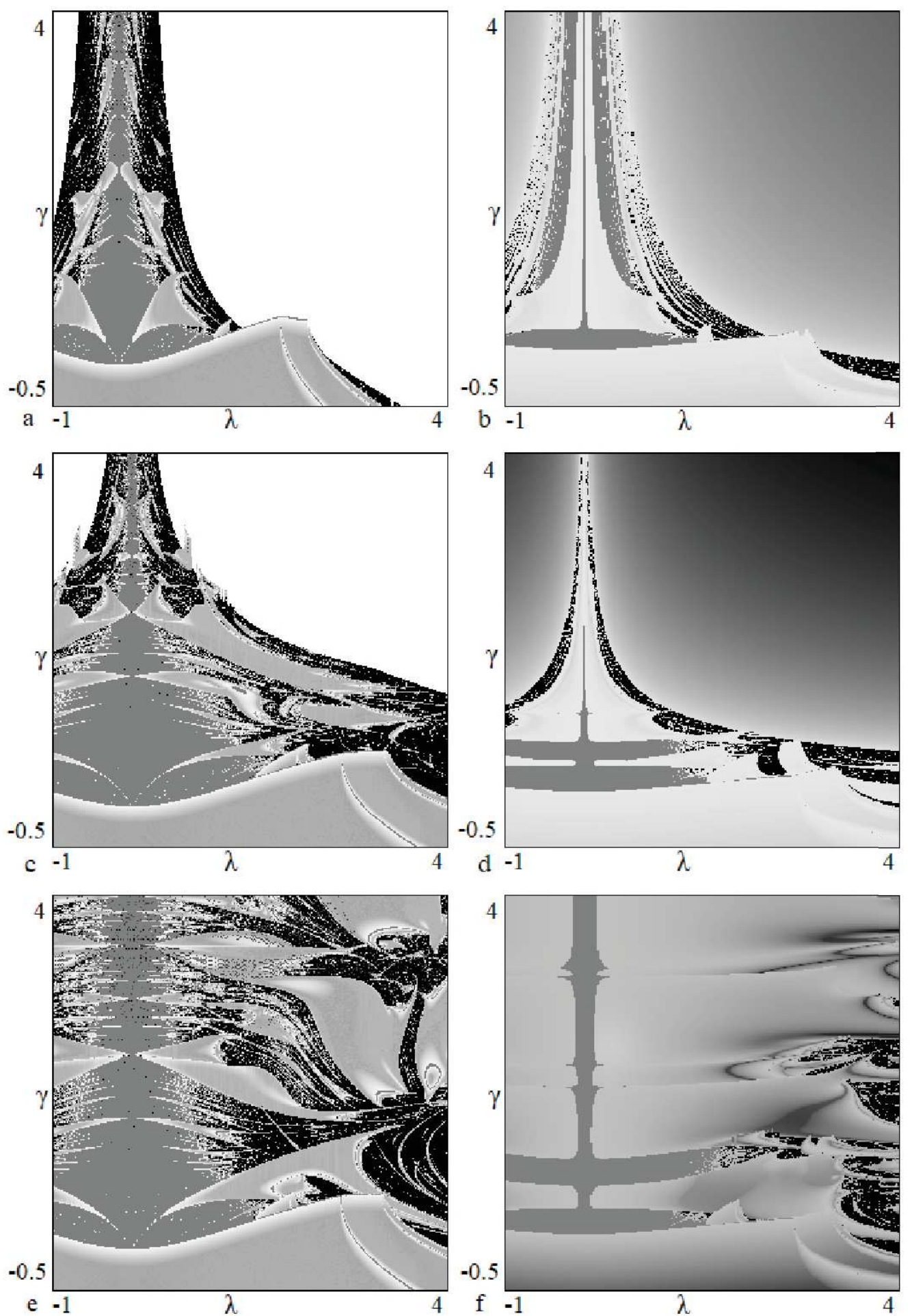

Рис. 1. $a, c, e-$ карты ляпуновских показателей для потоковой системы (1) для $F(x)=\lambda\left(1-x^{2} / 2\right)$, $F(x)=\lambda\left(1-x^{2} / 2+x^{4} / 24\right), F(x)=\lambda \cos x$, соответственно. $b, d, f$ - карты ляпуновских показателей для дискретного отображения (2). Цвета на картах ляпуновских показателей соответствуют различным режимам: оттенками серого показана область, соответствующая периодическому режиму, темно-серым показан квазипериодический режим, черным - хаотический, белым - область убегания траекторий на бесконечность. $\mu=0.5, \omega=4$

Fig. 1. $a, c, e-$ maps of the Lyapunov exponents for the flow system (1) for $F(x)=\lambda\left(1-x^{2} / 2\right)$, $F(x)=\lambda\left(1-x^{2} / 2+x^{4} / 24\right), F(x)=\lambda \cos x$, respectively. $b, d, f$-maps of the Lyapunov exponents for the discrete map (2). The colors on the maps of the Lyapunov exponents correspond to different regimes: grayscale for periodic, dark gray - quasi-periodic, black - chaotic, and the area of escape of trajectories to infinity is white. $\mu=0.5, \omega=4$ 
Можно заметить также, что часть языков синхронизации теперь связана с окнами периодичности в области хаоса, возникшего из каскада бифуркаций удвоения периода. В случае выбора функции внешнего воздействия $\cos x$ область разбегания на бесконечность исчезает полностью, а также заметно усложняется форма языков синхронизации при больших значениях $\gamma$.

\section{2. Устройство фазового пространства}

Отдельный интерес представляет исследование устройства фазового пространства потоковой системы (1). На рис. 2 приведены фазовые портреты при значениях параметра $\lambda$, соответствующих режиму периода 1 (рис. $2, a-c$ ), периода 2 (рис. $2, d, e$ ) и периода 4 (рис. $2, f$ ) в случае, когда нелинейная диссипация не является малой. Видно, что форма траекторий точек в фазовом пространстве в этом случае практически не зависит от вида функции внешнего воздействия.

При уменьшении нелинейной диссипации зависимость вида фазового пространства от функции внешнего воздействия становится явно заметной. На рис. 3 приведен набор фазовых портретов для последовательно уменьшающихся значений $\mu$ при фиксированных значениях остальных параметров, которые выбраны соответствующими квазипериодической области на картах динамических режимов. Как видно из рис. $3, a-c$, при больших значениях $\mu$ фазовые портреты для всех трех функций выглядят практически одинаково, однако с уменьшением $\mu$ они
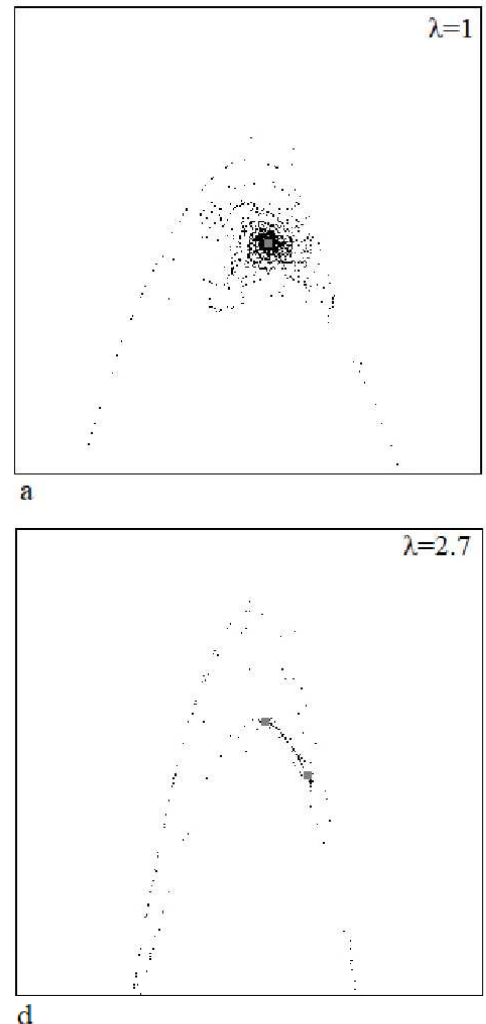
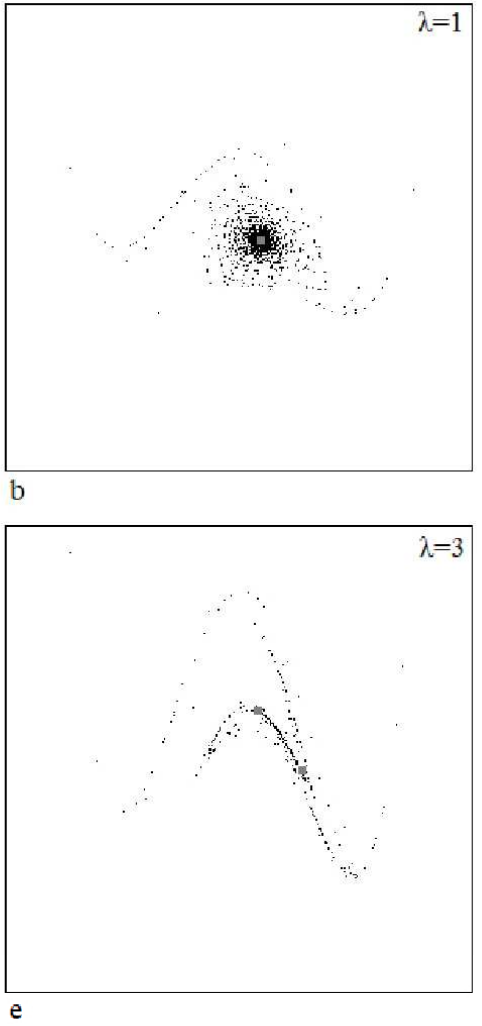
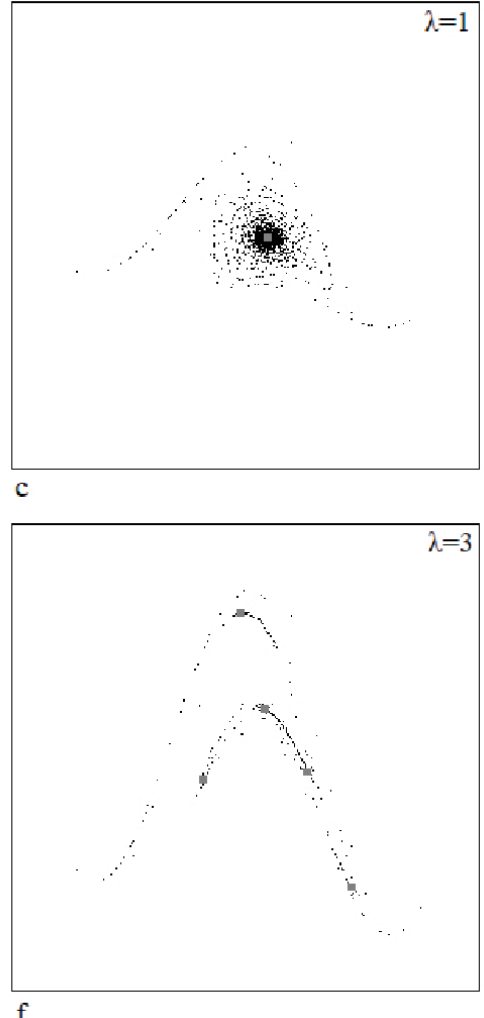

Рис. 2. Фазовые портреты, соответствующие следующим функциям: $a, d-F(x)=\lambda\left(1-x^{2} / 2\right) ; b, e-F(x)=$ $=\lambda\left(1-x^{2} / 2+x^{4} / 24\right) ; c, f-F(x)=\lambda \cos x ; \gamma=0.1, \mu=0.5$. По осям отложены координаты $x, \dot{x}$ в диапазоне $(-5 ; 5)$. Черным цветом показан переходный процесс, серым отмечены неподвижные устойчивые точки

Fig. 2. Phase portraits corresponding to: $a, d-F(x)=\lambda\left(1-x^{2} / 2\right) ; b, e-F(x)=\lambda\left(1-x^{2} / 2+x^{4} / 24\right)$; $c, f-F(x)=\lambda \cos x ; \gamma=0.1, \mu=0.5$. The range of coordinates $x, \dot{x}$ along the axes is $(-5 ; 5)$. Black color corresponds to transient process, fixed points are marked in gray color

Голоколенов А.B. 


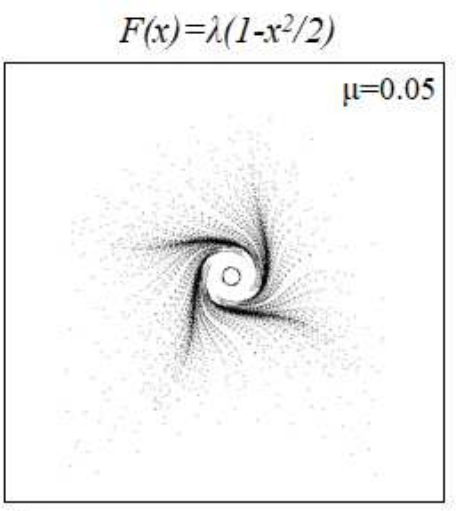

a
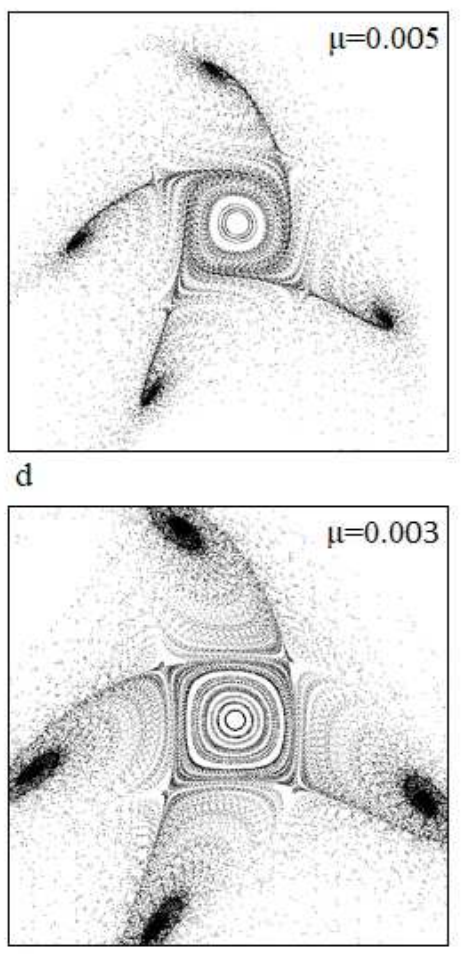

g

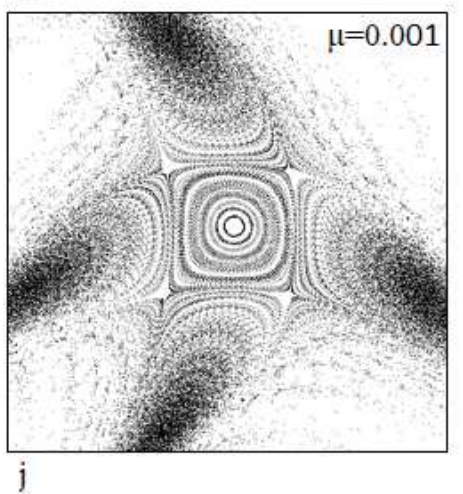

$F(x)=\lambda\left(1-x^{2} / 2+x^{4} / 24\right)$

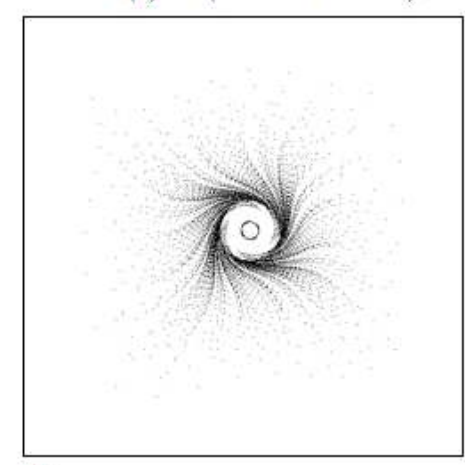

b
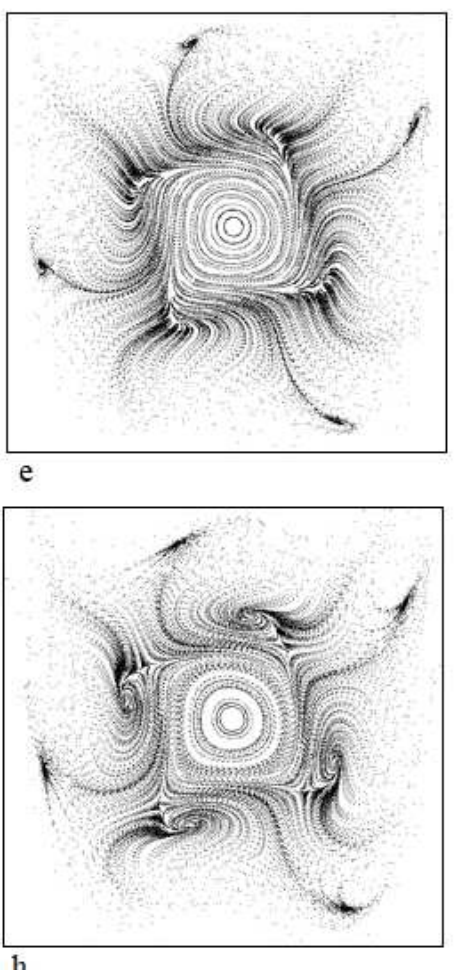

h

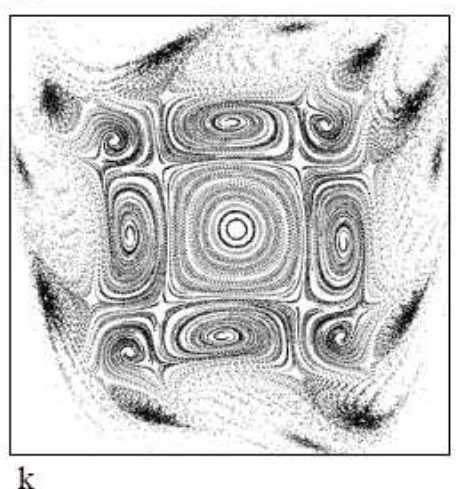

k

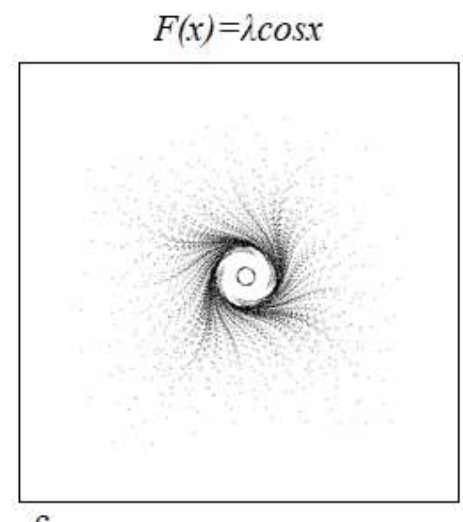

c

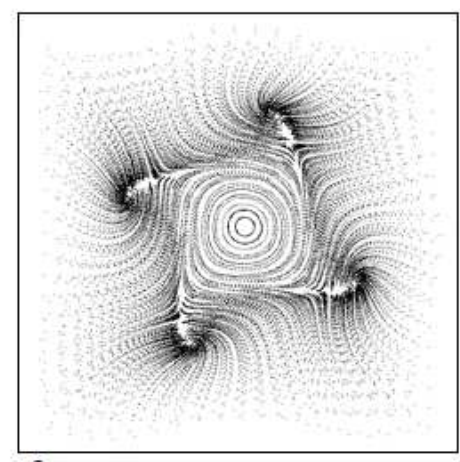

f
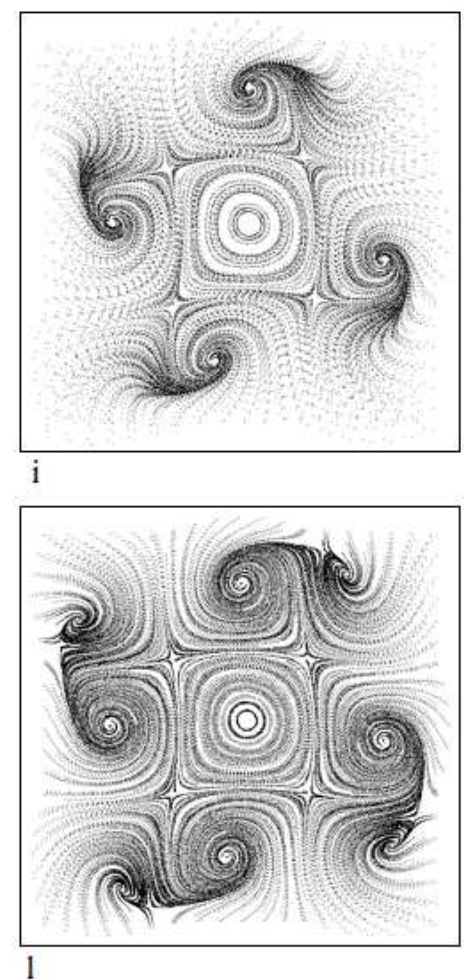

Рис. 3. Фазовые портреты для системы (1). Столбцы с рисунками соответствуют разным функциям внешнего воздействия, строки - разным параметрам нелинейной диссипации $\mu . \gamma=0.001, \lambda=0.3$. Для рисунков $a-o$ по осям отложены координаты $x, \dot{x}$ в диапазоне $(-5 ; 5)$

Fig. 3. Phase portraits for the system (1). The columns with figures correspond to different functions of external force, the lines - to different parameters of nonlinear dissipation $\mu . \gamma=0.001, \lambda=0.3$. For figures $a-o$ the range of coordinates $x, \dot{x}$ along the axes is $(-5 ; 5)$ 

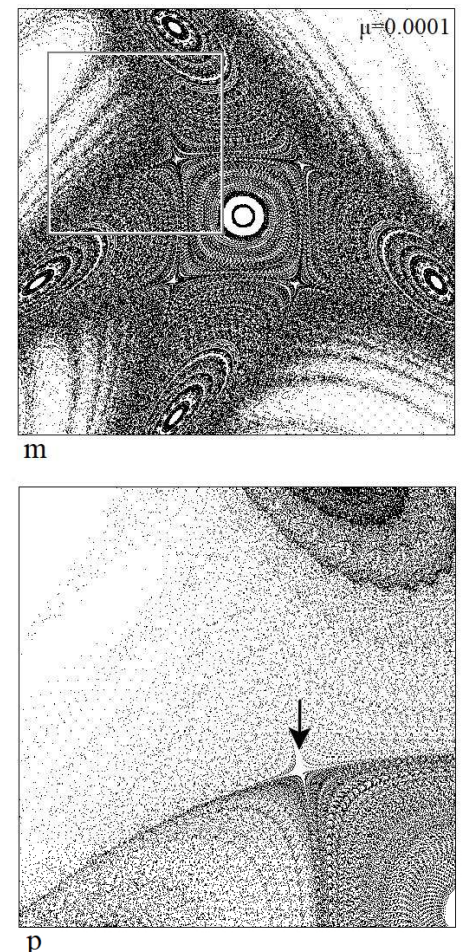

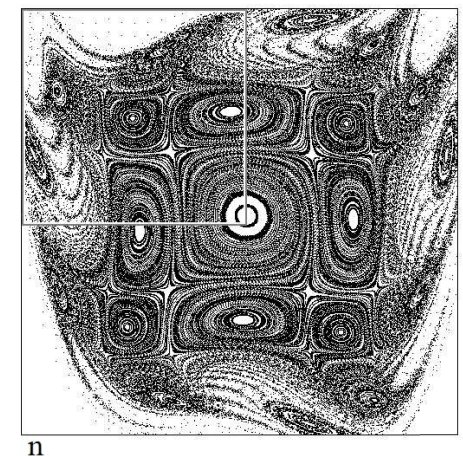

n

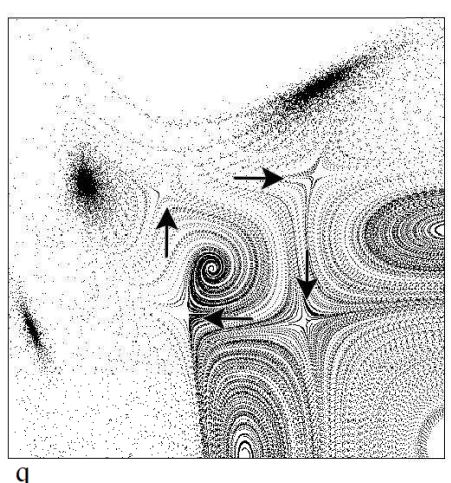

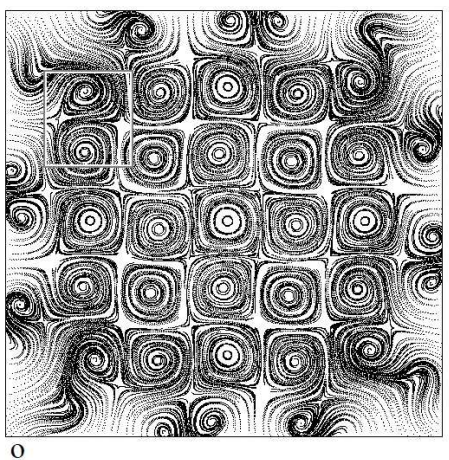

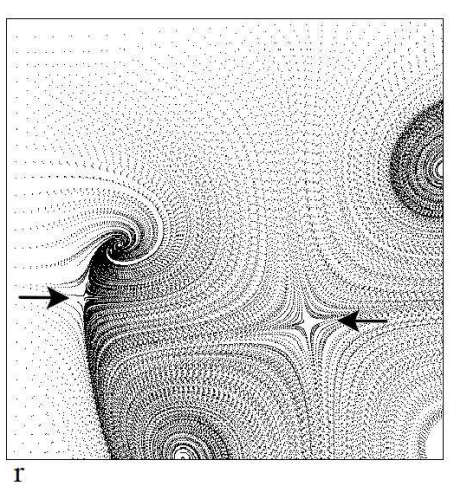

Рис. 3. (Продолжение) $p, q, r$ - увеличенные участки фазовой плоскости, соответствующие рис. $m, n, o$

Fig. 3. (Continuation) $p, q, r$ are the enlarged fragments of the phase space, corresponding to fig. $m, n, o$

«накапливают» различия, и общий вид фазовых портретов усложняется. В частности, в результате последовательности седло-узловых бифуркаций появляются устойчивые неподвижные точки, сосуществующие с расположенным в окрестности начала координат «основным» аттрактором. На рис. 3, $p-r$ приведены увеличенные фрагменты фазовых портретов (рис. $3, m-o$ ), иллюстрирующие более детально структуру фазового пространства в окрестности неподвижных точек. Так, на них хорошо видны объекты, траектории к которым притягиваются вдоль устойчивого направления и отдаляются вдоль неустойчивого (на рисунке они показаны стрелками) - седловые точки, по появлению которых можем судить о том, что произошла седло-узловая бифуркация.

Известно, что для консервативной системы (1) с функцией внешнего воздействия вида $F(x)=\lambda \cos x$ наблюдается стохастическая паутина [22]. Соответственно, в диссипативном случае количество седло-узловых бифуркаций будет увеличиваться по мере приближения к консервативному пределу, то есть уменьшения значения параметра диссипации. Так, на рис. 3, o представлен фазовый портрет для системы с функцией внешнего воздействия вида $F(x)=\lambda \cos x$ в случае, близком к консервативному. Количество бифуркаций в этом случае, как можно видеть, довольно велико, а структура фазового пространства закономерно похожа на стохастическую паутину. При переходе к разложению функции $F(x)$ в ряд Тейлора количество седлоузловых бифуркаций уменьшается с понижением порядка разложения в ряд. Так, для $F(x)=$ $=\lambda\left(1-x^{2} / 2+x^{4} / 24\right)$ с уменьшением $\mu$ новые седло-узловые бифуркации перестают происходить, и при достижении определенного значения $\mu$ структура фазового пространства перестает существенно меняться (ср. рис. $3, k, n)$. В случае же с $F(x)=\lambda\left(1-x^{2} / 2\right)$ происходит всего одна седло-узловая бифуркация, приводящая к появлению одного устойчивого цикла периода 4 (рис. $3, d$ ). На рис. 4 приведены фазовые портреты, иллюстрирующие процесс усложнения структуры фазового пространства для системы с $F(x)=\lambda\left(1-x^{2} / 2+x^{4} / 24\right)$ более подробно. Для этого подбирались значения параметра $\mu$ таким образом, чтобы полученные фазовые портре-

Голоколенов А.B. 

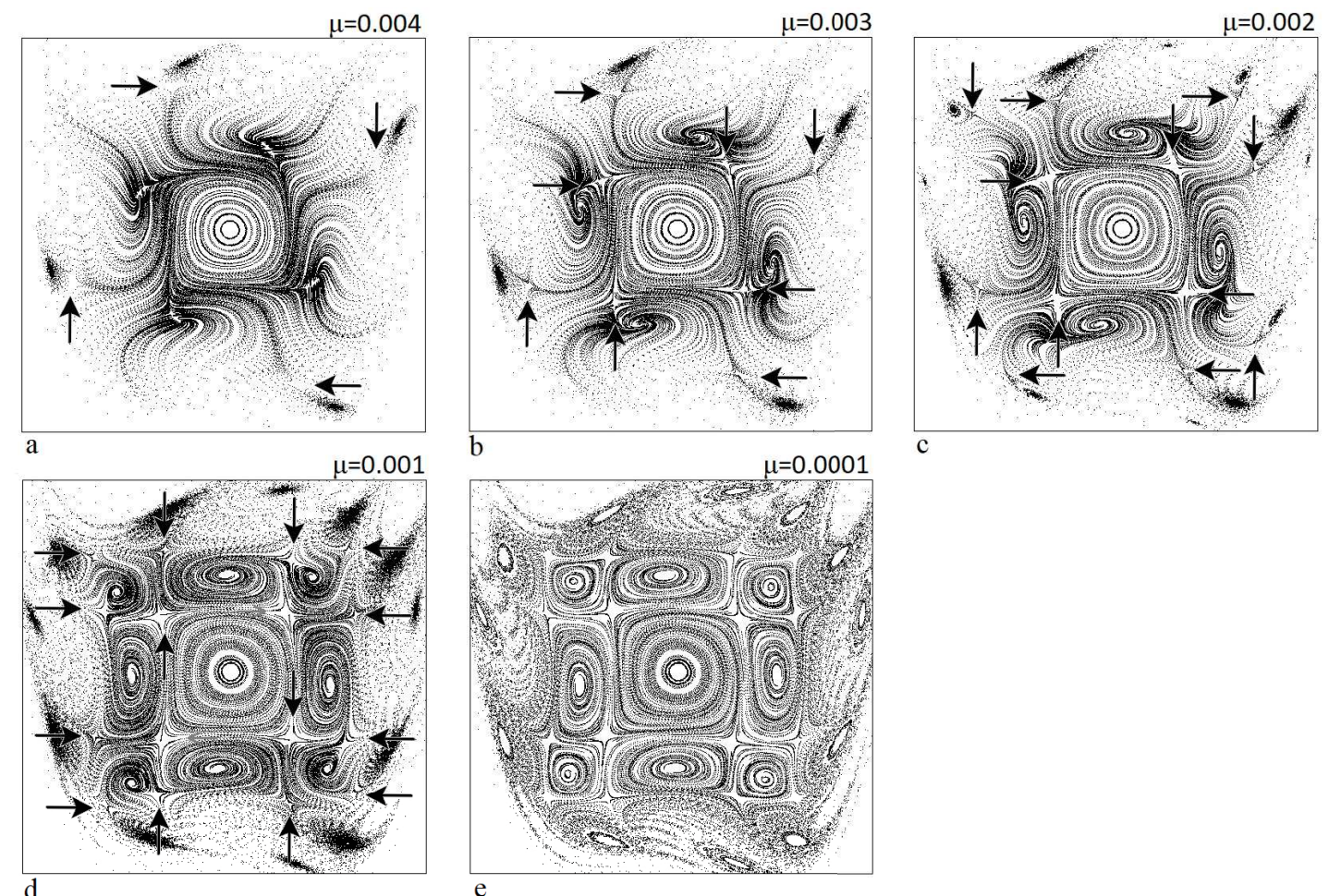

Рис. 4. Фазовые портреты для системы (1) для $F(x)=\lambda\left(1-x^{2} / 2+x^{4} / 24\right)$ при различных значениях $\mu$. Значения $\mu$ соответствуют моментам появления седел, которые на рисунках показаны стрелками. $\lambda=0.3$. По осям отложены координаты $x, \dot{x}$ в диапазоне $(-5 ; 5)$

Fig. 4. Phase portraits for system (1) for $F(x)=\lambda\left(1-x^{2} / 2+x^{4} / 24\right)$ for different values of $\mu$. The values of $\mu$ correspond to the moments of appearance of saddles, which are marked in the figures with arrows. $\lambda=0.3$. The range of coordinates $x$, $\dot{x}$ along the axes is $(-5 ; 5)$
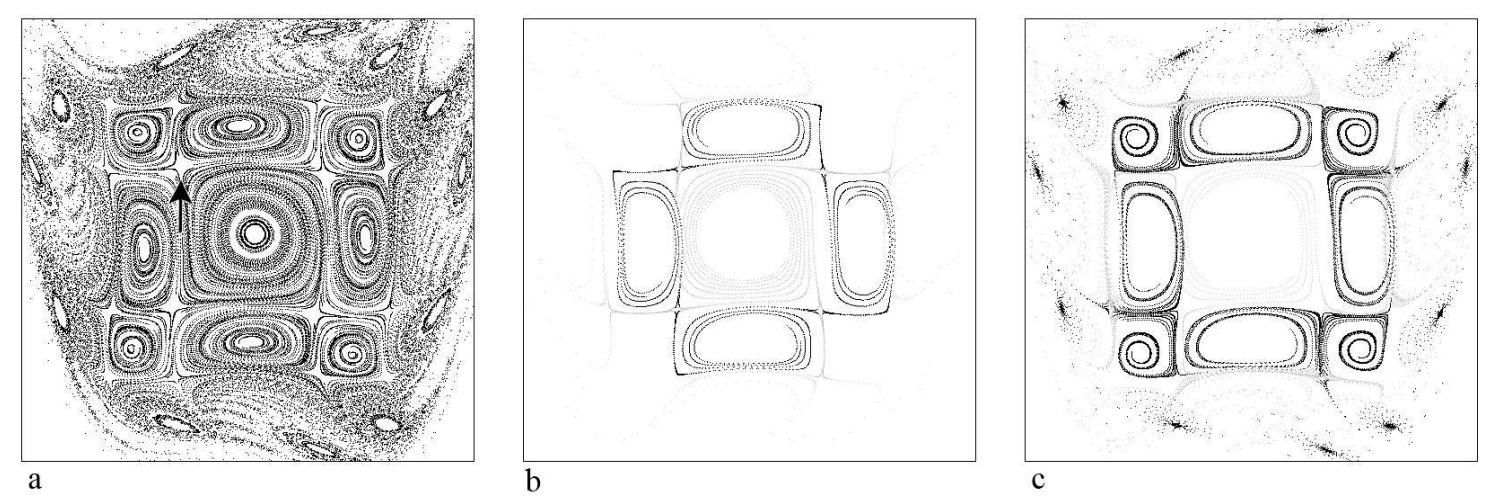

Рис. 5. $a$ - фазовый портрет для системы (1) для $F(x)=\lambda\left(1-x^{2} / 2+x^{4} / 24\right), b-$ многообразие для одного из седел, $c$ - многообразия для четырех различных седловых циклов, наложенные друг на друга. $\mu=0.0005, \lambda=0.3$, $\gamma=0.001$. Серым показаны устойчивые многообразия, черным - неустойчивые. По осям отложены координаты $x, \dot{x}$ в диапазоне $(-5 ; 5)$

Fig. 5. $a$ is the phase portrait for system (1) for $F(x)=\lambda\left(1-x^{2} / 2+x^{4} / 24\right), b$ is the manifold for one of the saddles, $c$ is the manifold for four different saddle cycles superimposed on each other $\mu=0.0005, \lambda=0.3, \gamma=0.001$. Gray color corresponds to stable manifolds, black - unstable. The range of coordinates $x, \dot{x}$ along the axes is $(-5 ; 5)$ 
ты соответствовали ситуации сразу после появления новых пар «седло - устойчивая точка», по которым мы определяем, что произошла седло-узловая бифуркация.

Для более детального изучения структуры фазового пространства также были построены устойчивые и неустойчивые многообразия точек, которые мы по результатам визуального анализа структуры фазового портрета определили как седловые (рис. 5). Для этого брались наборы начальных условий в окрестности одного из седел (на рис. 5, $а$ показано стрелкой), после чего система итерировалась в прямом и обратном времени. В результате были получены трансверсально пересекающиеся многообразия, проходящие через 4 неподвижные точки (рис. 5, б), что позволяет сделать вывод о наличии седлового цикла периода 4.

\section{Заключение}

В данной работе проведено исследование динамики находящейся под внешним импульсным воздействием автоколебательной системы с малым уровнем диссипации. Функция, описывающая зависимость амплитуды внешнего воздействия от динамической переменной, изменялась таким образом, чтобы осуществить переход от системы, для которой ранее [6-8] было показано наличие критического поведения гамильтоновского типа, к системе, демонстрирующей в консервативном пределе стохастическую паутину. Были построены карты динамических режимов и карты ляпуновских показателей при различных значениях нелинейной диссипации. При усложнении функции как для исходной потоковой системы, так и для приближенного дискретного отображения, происходит расширение областей хаотической динамики и квазипериодики на плоскости параметров. В результате область, в которой предполагается наличие критической точки Н-типа, оказывается внутри хаотической области - в отличие от ранее исследованного предельного случая, когда критическая точка Н-типа располагалась на границе двух областей хаотической динамики, возникших разными путями. Структура фазового пространства при значениях параметра нелинейной диссипации, не являющихся малыми, практически не зависит от функции внешнего воздействия. С уменьшением значения этого параметра общий вид фазового пространства усложняется - в результате последовательности седло-узловых бифуркаций рождаются сосуществующие с основным периодические устойчивые циклы. При этом для полиномиальных функций амплитуды внешнего воздействия число бифуркаций ограничено, и при достижении определенного уровня нелинейной диссипации вид фазового пространства перестает меняться. Более высокой степени полинома при этом соответствует большее количество седло-узловых бифуркаций. В случае же выбора функции $F(x)=\lambda \cos x$, соответствующей наличию в консервативном пределе стохастической паутины, с уменьшением параметра нелинейной диссипации число бифуркаций растет, по-видимому, неограниченно.

\section{Библиографический список}

1. Шустер Г. Детерминированный хаос. М.: Мир, 1990, 240 с.

2. Райхл Л.Е. Переход к хаосу в консервативных классических и квантовых системах. М.: ИКИ; Ижевск: РХД, 2008, 756 с.

3. Zisook A.B. Universal effects of dissipation in two-dimensional mappings // Physical Review A. 1982. T. 24, № 3. P. 1640-1642.

4. Морозов А.Д. Резонансы, циклы и хаос в квазиконсервативных системах. Серия: Современная математика. М.; Ижевск: РХД, 2005. 424 с.

5. Kuznetsov S.P., Kuznetsov A.P., Sataev I.R. Multiparameter critical situations, universality and

Голоколенов А.В.

Известия вузов. ПНД, 2019, т. 27, № 3 
scaling in two-dimensional period-doubling maps // Journal of Statistical Physics. 2005. T. 121, № 5-6. P. 697-748.

6. Кузнеиов А.П., Кузнеиов С.П., Савин А.В., Савин Д.В. Автоколебательная система с компенсируемой диссипацией: Динамика дискретной модели // Известия вузов. Прикладная нелинейная динамика. 2008. Т. 16, № 5. С. 127-138.

7. Кузнецов А.П., Кузнецов С.П., Савин А.В., Савин Д.В. О возможности реализации в автоколебательной системе с внешним периодическим воздействием универсального поведения, характерного для перехода к хаосу через удвоения периода в консервативных системах // Письма в ЖТФ. 2008. Т. 34, вып. 22. С. 72-80.

8. Savin D.V., Savin A.V., Kuznetsov A.P., Kuznetsov S.P., Feudel U. The self-oscillating system with compensated dissipation - the dynamics of the approximate discrete map // Dynamical Systems: An International Journal. 2012. Vol. 27. P. 117-129.

9. Feudel U., Grebogi C., Hunt B.R., Yorke J.A. Map with more than 100 coexisting low-period periodic attractors // Physical Review E. 1996. Vol. 54, no. 1. P. 71-81.

10. Feudel U., Grebogi C., Poon L., Yorke J.A. Dynamical properties of a simple mechanical system with a large number of coexisting periodic attractors // Chaos, Solitons \& Fractals. 1998. Vol.9, no. 1-2. P. 171-180.

11. Blazejczyk-Okolewska B., Kapitaniak T. Coexisting attractors of impact oscillator // Chaos, Solitons \& Fractals. 1998. Vol. 9, no. 8. P. 1439-1443.

12. Feudel U., Grebogi $C$. Why are chaotic attractors rare in multistable systems? // Physical Review Letters. 2003. Vol. 91, no. 13. 134102.

13. de Freitas A.S.T., Viana R.L., Grebogi C. Multistability, basin boundary structure, and chaotic behavior in a suspension bridge model // International Journal of Bifurcation and Chaos. 2004. Vol. 14, no. 3. P. 927-950.

14. Rech P., Beims M., Gallas J. Basin size evolution between dissipative and conservative limits // Physical Review E. 2005. Vol. 71, no. 1. 017202.

15. Колесов А.Ю., Розов Н.Х. О природе явления буферности в слабо диссипативных системах // Теоретическая и математическая физика. 2006. Т. 146, № 3. С. 447-466.

16. Кузнеиов А.П., Савин А.В., Савин Д.В. Отображение Икеды: от диссипативного к консервативному случаю // Известия вузов. ПНД. 2006. Т. 14, № 2. С. 94-106.

17. Кузнецов А.П., Савин А.В., Савин Д.В. Особенности динамики почти консервативного отображения Икеды // Письма в ЖТФ. 2007. Т. 33, вып. 3. С. 57-63.

18. Kuznetsov A.P., Savin A.V., Savin D.V. On some properties of nearly conservative dynamics of Ikeda map // Nonlinear Phenomena in Complex Systems. 2007. Vol. 10, no. 4. P. 393-400.

19. Feudel U. Complex dynamics in multistable systems // International Journal of Bifurcation and Chaos. 2008. Vol. 18, no. 6. P. 1607-1626.

20. Kuznetsov A.P., Savin A.V., Savin D.V. On some properties of nearly conservative dynamics of Ikeda map and its relation with the conservative case // Physica A. 2008. Vol. 387. no. 7. P. 1464-1474.

21. Мозер Ю. КАМ-теория и проблемы устойчивости. М.; Ижевск: РХД, 2001. 448 с.

22. Заславский Г.М. Стохастичность динамических систем. М.: Наука, 1984. 272 с.

23. Савин А.В., Савин Д.В. Структура бассейнов притяжения сосуществующих аттракторов слабодиссипативного «отображения-паутины»// Нелинейный Мир. 2010. Т. 8, № 2. C. $70-71$. 
24. Фельк E.B. Влияние слабой нелинейной диссипации на структуры типа «стохастическая паутина» // Известия вузов. Прикладная нелинейная динамика. 2013. Т. 21, № 3. С. 72-79.

25. Felk E.V., Kuznetsov A.P., Savin A.V. Multistability and transition to chaos in the degenerate Hamiltonian system with weak nonlinear dissipative perturbation // Physica A. 2014. T. 410. P. 561-572.

\section{References}

1. Schuster H.G., Just W. Deterministic Chaos, WILEY-VCH Verlag, Weinheim, 2005, 287 p.

2. Reichl L.E. The Transition to Chaos in Conservative Classical Systems: Quantum Manifestations. New York: Springer-Verlag, 1992, $551 \mathrm{p}$.

3. Zisook A.B. Universal effects of dissipation in two-dimensional mappings. Physical Review A, 1982, vol. 24, no. 3, pp. 1640-1642.

4. Morozov A.D. Resonances, Cycles and Chaos in Quasi-Conservative Systems, Moscow; Izhevsk: Regular and Chaotic Dynamics, 2005, 424 p. (in Russian).

5. Kuznetsov S.P., Kuznetsov A.P., Sataev I.R. Multiparameter critical situations, universality and scaling in two-dimensional period-doubling maps. Journal of Statistical Physics, 2005, vol. 121, no. 5-6, pp. 697-748.

6. Kuznetsov A.P., Kuznetsov S.P., Savin A.V., Savin D.V. Autooscillating system with compensated dissipation: Dynamics of approximate discrete map. Izvestiya VUZ. Applied Nonlinear Dynamics, 2008, vol. 16, no. 5, pp. 127-138 (in Russian).

7. Kuznetsov A.P., Kuznetsov S.P., Savin A.V., Savin D.V. On the possibility for an autooscillatory system under external periodic drive action to exhibit universal behavior characteristic of the transition to chaos via period-doubling bifurcations in conservative systems. Technical Physics Letters, 2008, vol. 34, pp. 985-988.

8. Savin D.V., Savin A.V., Kuznetsov A.P., Kuznetsov S.P., Feudel U. The self-oscillating system with compensated dissipation: Dynamics of approximated discrete map. Dynamical Systems: An International Journal, 2012, vol. 27, pp. 117-129.

9. Feudel U., Grebogi C., Hunt B.R., Yorke J.A. Map with more than 100 coexisting low-period periodic attractors. Physical Review E, 1996, vol. 54, no. 1, pp. 71-81.

10. Feudel U., Grebogi C., Poon L., Yorke J.A. Dynamical properties of a simple mechanical system with a large number of coexisting periodic attractors. Chaos, Solitons \& Fractals, 1998, vol. 9, pp. 171-180.

11. Blazejczyk-Okolewska B., Kapitaniak T. Coexisting attractors of impact oscillator. Chaos, Solitons \& Fractals, 1998, vol. 9, no. 8, pp. 1439-1443.

12. Feudel U., Grebogi C. Why are chaotic attractors rare in multistable systems? Physical Review Letters, 2003, vol. 91, no. 13, 134102.

13. de Freitas A.S.T., Viana R.L., Grebogi C. Multistability, basin boundary structure, and chaotic behavior in a suspension bridge model. International Journal of Bifurcation and Chaos, 2004, vol. 14, no. 3, pp. 927-950.

14. Rech P., Beims M., Gallas J. Basin size evolution between dissipative and conservative limits. Physical Review E, 2005, vol. 71, no. 1, 017202.

15. Kolesov A.Yu., Rozov N.Kh., The nature of the bufferness phenomenon in weakly dissipative systems. Theoret. and Math. Phys., 2006, vol. 146, no. 3, pp. 376-392.

16. Kuznetsov A.P., Savin A.V., Savin D.V. Ikeda map: From a dissipative to conservative cases of

Голоколенов A.B.

Известия вузов. ПНД, 2019, т. 27, № 3 
the Ikeda map. Izvestiya VUZ. Applied Nonlinear Dynamics, 2006, vol. 14, no. 2, pp. 94-106 (in Russian).

17. Kuznetsov A.P., Savin A.V., Savin D.V. Features in dynamics of an almost conservative Ikeda map. Technical Physics Letters, 2007, vol. 33, iss. 2, pp. 122-124.

18. Kuznetsov A.P., Savin A.V., Savin D.V. On some properties of nearly conservative dynamics of Ikeda map. Nonlinear Phenomena in Complex Systems, 2007, vol. 10, no. 4, pp. 393-400.

19. Feudel U. Complex dynamics in multistable systems. International Journal of Bifurcation and Chaos, 2008, vol. 18, no. 6, pp. 1607-1626.

20. Kuznetsov A.P., Savin A.V., Savin D.V. On some properties of nearly conservative dynamics of Ikeda map and its relation with the conservative case. Physica A, 2008, vol. 387, no. 7, pp. 1464-1474.

21. Moser J. KAM-Theory and Stability Problems. Moscow; Izhevsk: Regular and Chaotic Dynamics, 2001, 448 p. (in Russian).

22. Zaslavsky G.M. Stochasticity of Dynamical Systems. Moscow: Nauka, 1984, 272 p. (in Russian).

23. Savin A.V., Savin D.V. Structure of attraction basins of coexisting attractors of weakly dissipative «web-map». Nelinejnii Mir, 2010, vol. 8, no. 2, pp. 70-71 (in Russian).

24. Felk E.V. The effect of weak nonlinear dissipation on structures of the «stochastic web» type. Izvestiya VUZ. Applied Nonlinear Dynamics, 2013, vol. 21, no. 3, pp. $72-79$ (in Russian).

25. Felk E.V., Kuznetsov A.P., Savin A.V. Multistability and transition to chaos in the degenerate Hamiltonian system with weak nonlinear dissipative perturbation. Physica A, 410, 2014, pp. 561-572.

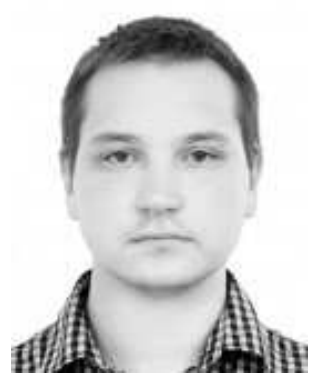

Голоколенов Александр Владимирович - родился в Саратове (1995). Окончил Саратовский государственный университет (2017). Защитил бакалаврскую работу по теме «Аттракторы слабодиссипативного стандартного отображения с малыми бассейнами притяжения». В настоящее время заканчивает обучение в магистратуре факультета нелинейных процессов СГУ. Имеет публикации в сборниках материалов конференций. Область научных интересов - динамика нелинейных систем с малым уровнем диссипации.

Россия, 410012 Саратов, ул. Астраханская, 83

Саратовский национальный исследовательский государственный университет

E-mail: golokolenovav@gmail.com 